\title{
Crystal orientation measurements using SEM-EBSD under unconventional conditions
}

\author{
Karsten Kunze $e^{\text {a) }}$ \\ ETH Zurich, Scientific Center for Optical and Electron Microscopy (ScopeM), Zurich, Switzerland
}

(Received 9 March 2015; accepted 12 March 2015)

Electron backscatter diffraction (EBSD) is a micro-analytical technique typically attached to a scanning electron microscope (SEM). The vast majority of EBSD measurements is applied to planar and polished surfaces of polycrystalline bulk specimen. In this paper, we present examples of using EBSD and energy-dispersive X-ray spectroscopy (EDX) to analyze specimens that are not flat, not planar, or not bulk - but pillars, needles, and rods. The benefits of low vacuum SEM operation to reduced drift problems are displayed. It is further demonstrated that small and thin specimens enhance the attainable spatial resolution for orientation mapping (by EBSD or transmission Kikuchi diffraction) as well as for element mapping (by EDX). ( $) 2015$ International Centre for Diffraction Data.

[doi:10.1017/S0885715615000263]

Key words: electron backscatter diffraction, transmission Kikuchi diffraction, energy dispersive

$\mathrm{X}$-ray spectroscopy, low vacuum mode

\section{INTRODUCTION}

Electron backscatter diffraction (EBSD) is a microanalytical technique typically attached to a scanning electron microscope (SEM). It provides information on the crystallographic structure and phase, crystal orientation, and/or lattice strain from volumes in the size of $10-100 \mathrm{~nm}$
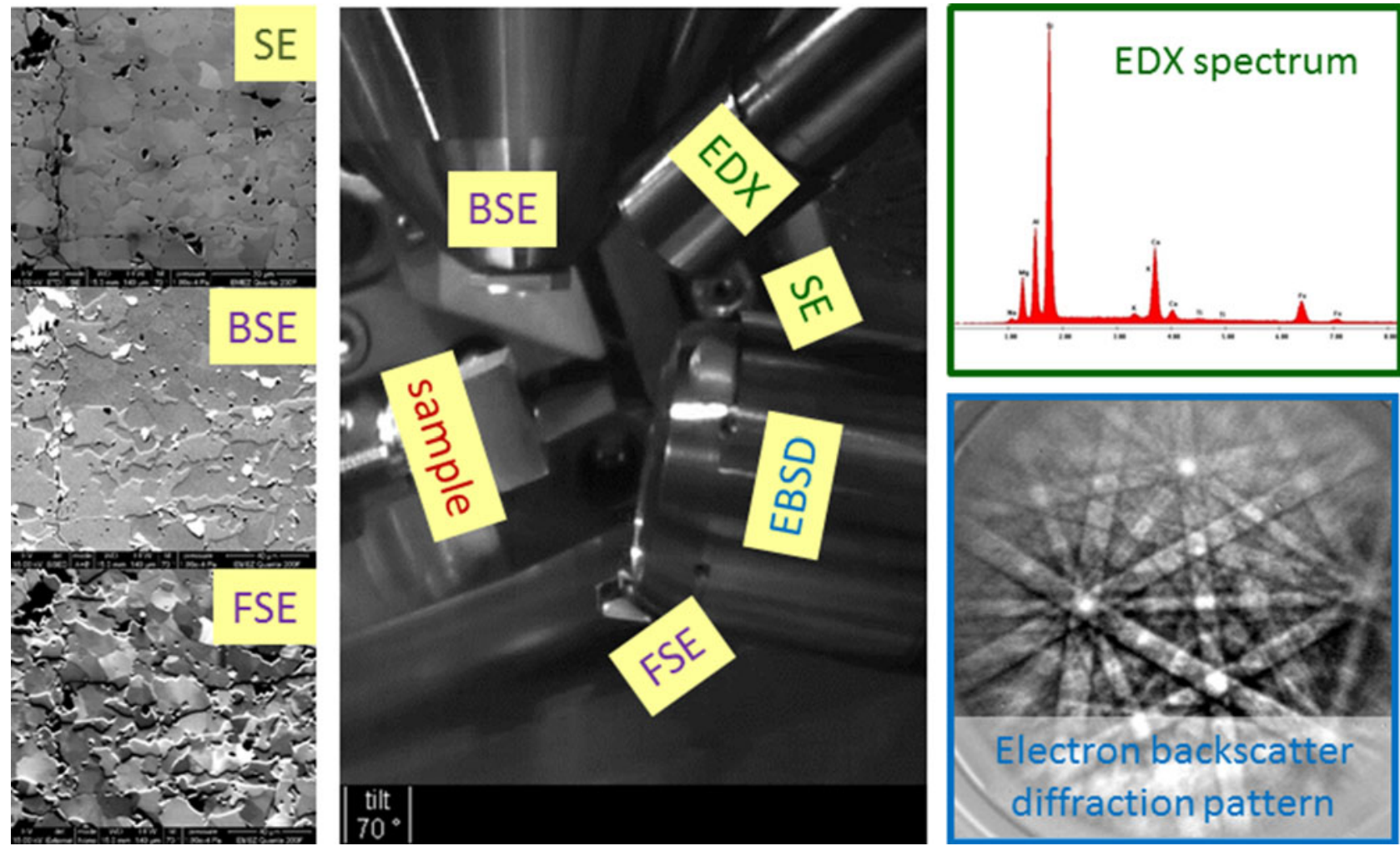

Figure 1. (Color online) Setup of an analytical SEM for simultaneous detection of SE, BSE, FSE imaging signals, EDX spectra, and EBSD patterns. Images of an interfingering contact between magnetite (brighter gray, bottom left in the BSE image) and hematite (darker gray, upper right in the BSE image) illustrate topographic, compositional, and orientation contrast (sample courtesy of Heinrich Siemes, Aachen).

\footnotetext{
${ }^{a}$ Author to whom correspondence should be addressed. Electronic mail: karsten.kunze@scopem.ethz.ch
} 


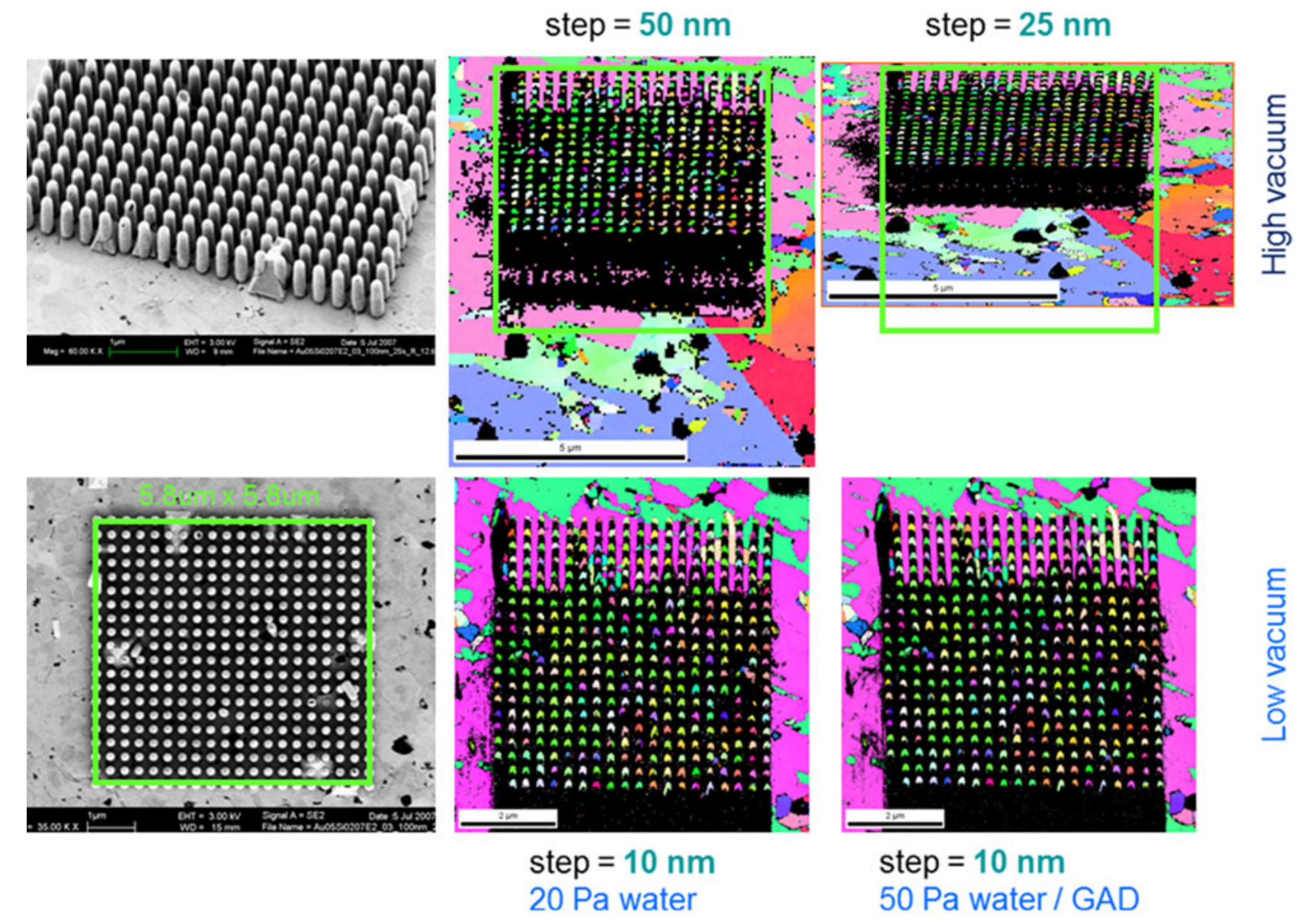

Figure 2. (Color online) Regular array of Gold pillars mapped in conventional EBSD geometry (substrate tilted by $70^{\circ}$ ), with major drift problems under high vacuum in the SEM (top), and negligible drift under low vacuum (Buzzi, 2009).

in diameter (Zaefferer, 2007). Automated sequential acquisition enables microstructural mapping, also called orientation imaging microscopy (OIM) (Adams et al., 1993).
As such it allows quantitative characterization and interpretation of the diverse multi-modal imaging from secondary electron (SE), backscatter electron (BSE) or forescatter electron (FSE) detector signals, giving rise to topographic,
$10 \mathrm{~Pa}$

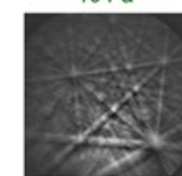

$50 \mathrm{~Pa}$

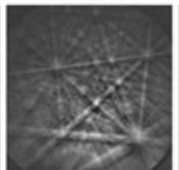

$100 \mathrm{~Pa}$

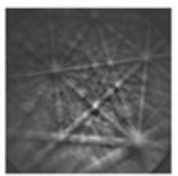

$150 \mathrm{~Pa}$

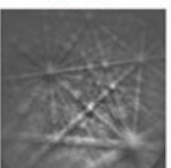

$200 \mathrm{~Pa}$
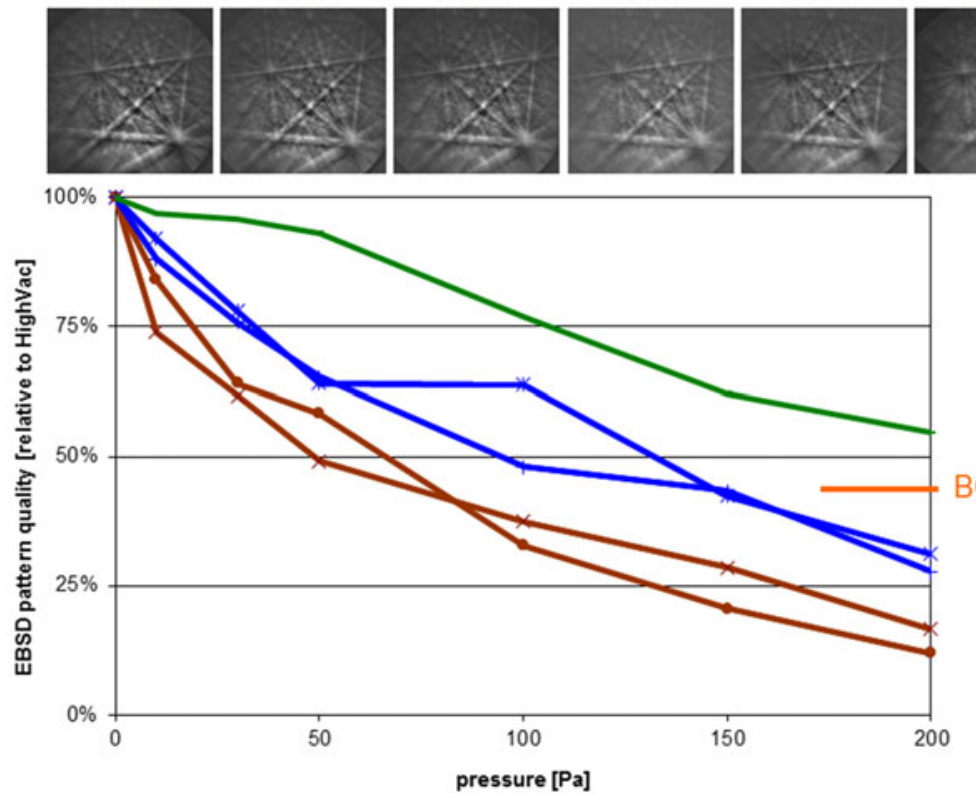

$300 \mathrm{~Pa}$
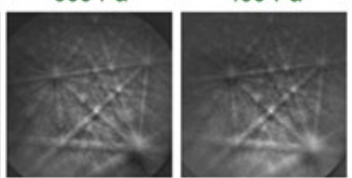

\section{Water+GAD}

BGPL $\frac{7 \mathrm{~mm}}{>15 \mathrm{~mm}}$

Water

Nitrogen

Figure 3. (Color online) Variation of the EBSD pattern quality with increasing SEM chamber pressure. Sequence of patterns from calcite (dynamic background corrected, top row); relative pattern quality (parameter IQ of OIM software, EDAX, USA) vs. chamber pressure. 

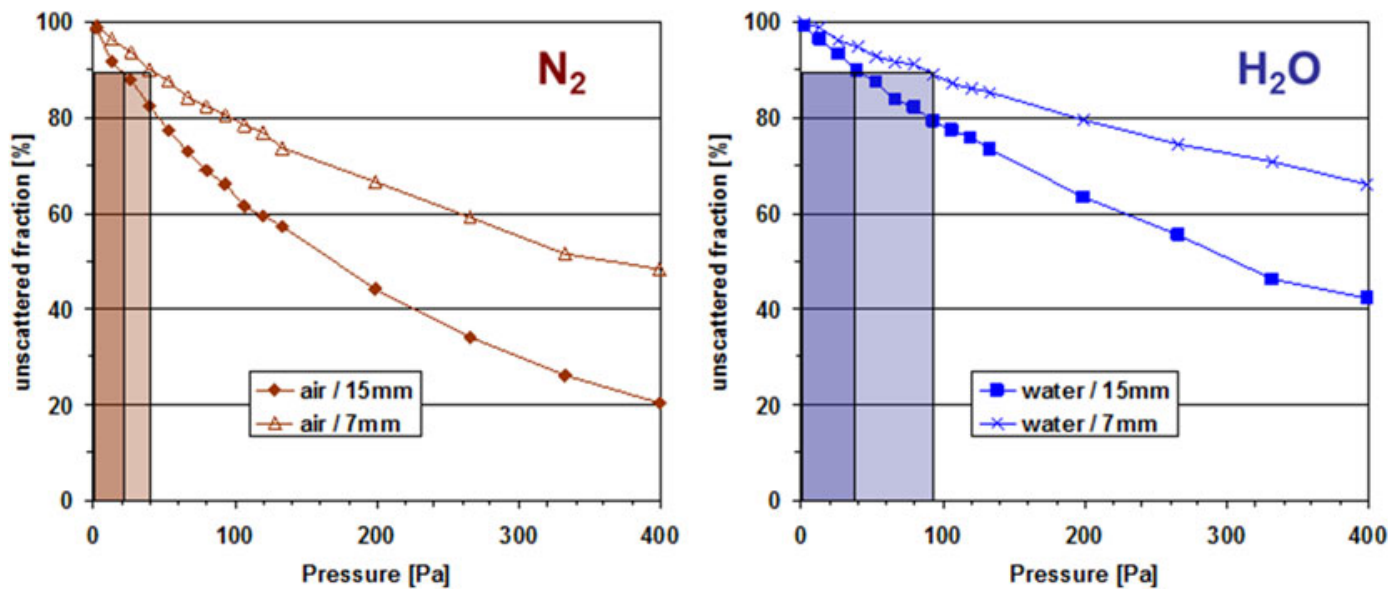

Figure 4. (Color online) Simulation of reduction of unscattered fraction of the electron beam with increasing SEM chamber pressure, facilitated by freeware Casino version 2.42 (Drouin et al., 2007). The amount scattering is predicted to reduce for shorter working distance (BGPL), water preferred to air, and lower pressure.

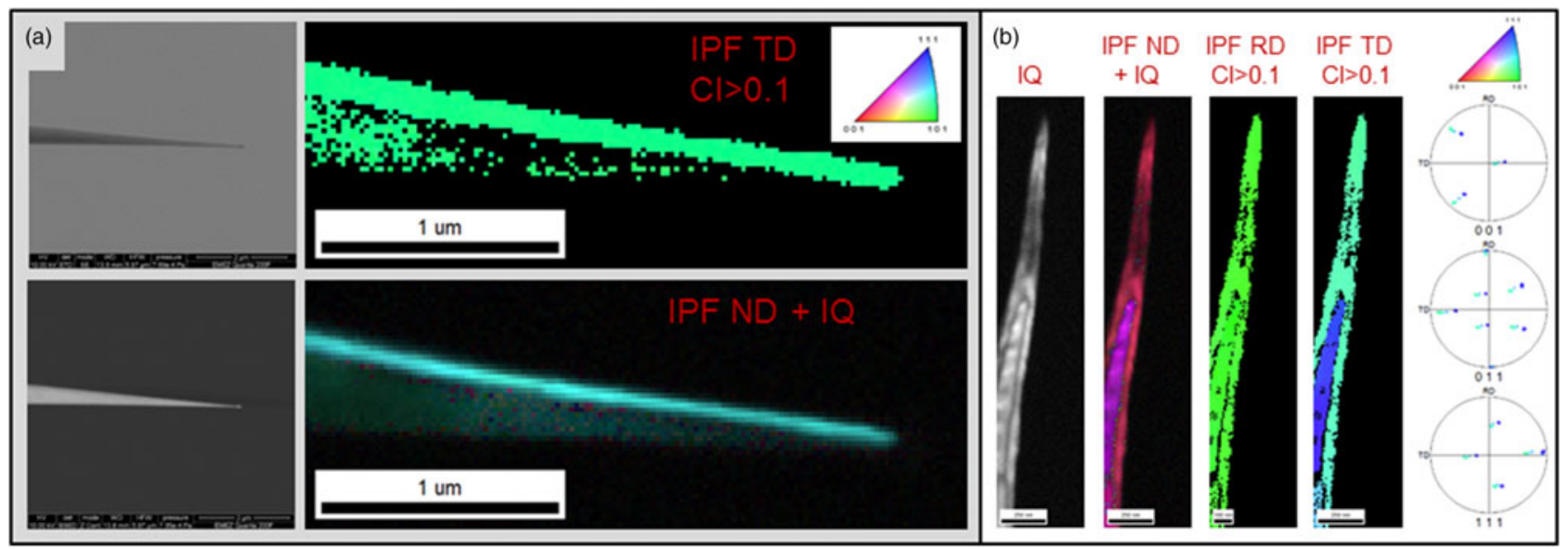

Figure 5. (Color online) Sharp tips of tungsten needles are analyzed for their crystal orientation. (a) In horizontal position, only a narrow strip is accessible to high-quality data. (b) In downwards tilted position, the needle is accessible in its full extent, and the mapping allows us to separate grain boundaries between raster steps of $10 \mathrm{~nm}$ (sample courtesy of Urs Ramsperger, Zürich).

compositional, and orientation contrast (Figure 1; Payton and Nolze, 2013). It is applied complementary to energydispersive X-ray spectroscopy (EDX or EDS), which provides information on elemental composition, typically at the micrometer scale. In that sense, EDX can be seen as the microanalytical equivalent to X-ray fluorescence, whereas EBSD stands as the micro-analytical pendant to X-ray diffraction.

The vast majority of EBSD measurements is applied to planar and polished surfaces of polycrystalline bulk specimen. In this paper, we present examples of using EBSD and EDX to analyze specimens that are not flat, not planar, or not bulk but pillars, needles, and rods.

\section{PILLARS: THE BENEFITS OF LOW VACUUM}

Sub-micrometer-sized Gold pillars (Buzzi, 2009) deliver good quality EBSD patterns from their top head area, while no indexable patterns are available from the interspace between pillars in a dense array (Figure 2). Despite of being electrically well-conductive, mapping of such arrays is destructed by major drift problems because of local charging under high vacuum in the SEM chamber. Drift is nearly absent in mappings under low vacuum (water vapor), and stability is further improved by the snorkel attachment of the gaseous analytical detector (GAD) reducing the beam gas path length (BGPL) between bottom pressure-limiting aperture and sample. Grain boundaries are possible to separate between raster steps as small as $10 \mathrm{~nm}$ (Kunze et al., 2008).

Any gaseous medium in the SEM vacuum chamber causes some scattering of the incident and diffracted beams, and thus should be avoided as much as possible. The amount of reduction in EBSD pattern quality can be minimized by shorter BGPL, lower pressure, and using water vapor instead of air, as demonstrated by the experimental data (Figure 3 ) as well as by Monto-Carlo simulations (Drouin et al., 2007) of unscattered beam fraction (Figure 4).

\section{NEEDLES: FINDING THE OPTIMUM MEASUREMENT GEOMETRY}

Instead of analyzing pillars from the top, they may be mounted with their axis perpendicular to the electron beam (Camus et al., 2011). Sharply etched tungsten tips were analyzed for the crystal orientation at the very front tip. With the needle 


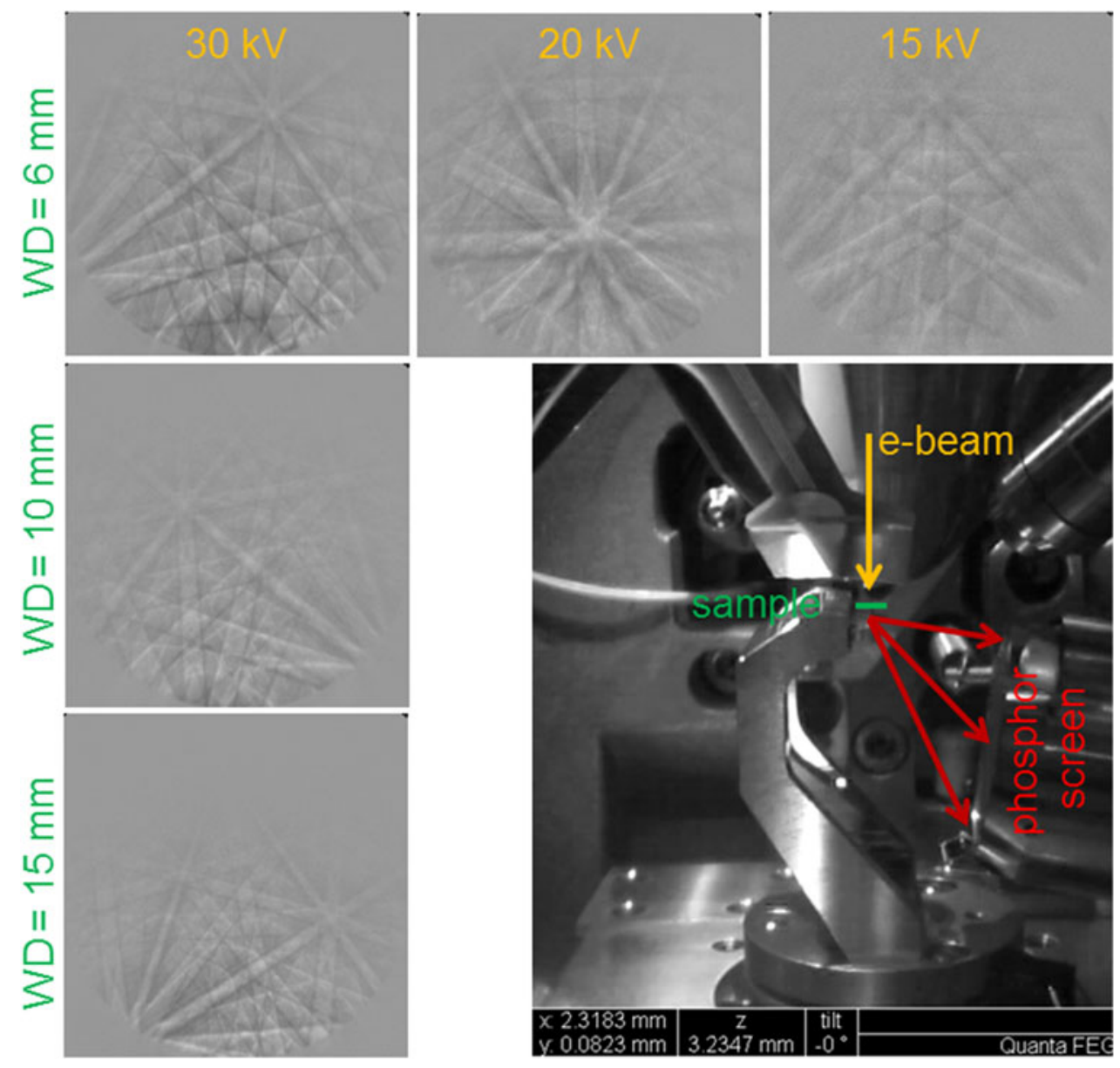

Figure 6. (Color online) Setup of a modified EBSD system to collect TKD patterns. Variation of (copper) pattern illumination with working distance (WD) and accelerating voltage (in $\mathrm{kV}$ ).

directed horizontally, a narrow strip on the conical surface satisfies about the $70^{\circ}$ tilt, which gives rise to high-quality EBSD patterns (Figure 5). Remarkably, reliable and valid indexations were also obtained from thinker parts of the cone.

The full extent of the needle is available to orientation mapping, if the needle is mounted dipping $55^{\circ}$ downwards to the EBSD detector. The grain boundary (of about $20^{\circ}$ misorientation angle) is resolved in mappings with raster steps of $10 \mathrm{~nm}$.

\section{RODS: USING THE TRANSMISSION KIKUCHI DIFFRACTION (TKD) TECHNIQUE}

It was proposed recently (Keller and Geiss, 2012; Trimby, 2012), how to covert the EBSD system from backscatter to transmission geometry. With a suitable holder (here the commercially available holder for scanning transmission electron microscopy) for thin specimens, the EBSD detector can collect TKD patterns (Figure 6) from electron transparent foils conventionally prepared for transmission electron microscopy (TEM) (e.g. Kunze et al., 2013). The phosphor screen is optimally illuminated for high accelerating voltage and short working distance.

$\mathrm{Fe}-\mathrm{Co}$ rods on sub-hundred nanometer size were sparsely dispersed on a carbon-foil TEM grid, and crystal orientation mapping was facilitated. A rough statistical overview is obtained, when the maps are post-processed for grain size and grain shape, and the color legend is adopted to display the crystal direction parallel to the long grain axis (Figure 7). This approach verifies $\langle 111\rangle$ to be preferred along the rod long axis.

Individual $\mathrm{Fe}-\mathrm{Co}$ nano-rods were mounted at the front tip of Si-cantilevers for orientation mapping (by TKD) as well as element mapping (by EDX); see Figure 8. The nano-rods contain subgrains with relevant misorientation angles, but are preferred aligned along $\langle 111\rangle$. Since the X-ray emission volume is kept small for such thin rods, the element maps reveal some remarkable spatial resolution well below $100 \mathrm{~nm}$, and a seed layer $(\mathrm{Pt})$ of $10-20 \mathrm{~nm}$ thickness is detected. Care must be taken when interpreting the abundant elements, as the maps also contain stray signals from the cantilever ( $\mathrm{Si}$ ), sample hol$\operatorname{der}(\mathrm{Al})$, and other parts in the sample surroundings.

\section{DISCUSSION AND CONCLUSIONS}

The presented examples demonstrate application fields of EBSD and EDX as micro- and nano-analytical tools for characterization of samples beyond the conventionally prepared flat, polished bulk specimens. It is shown that:

- Charging related drift problems may occur even on electrically well-conducting materials and are largely suppressed by introducing a water vapor in a low vacuum environment.

- Needles and pillars are best analyzed when placed in downwards tilted position.

- Nano-rods and other thin objects (TEM foils or lamellae) are efficiently analyzed by TKD and EDX in the SEM, both 

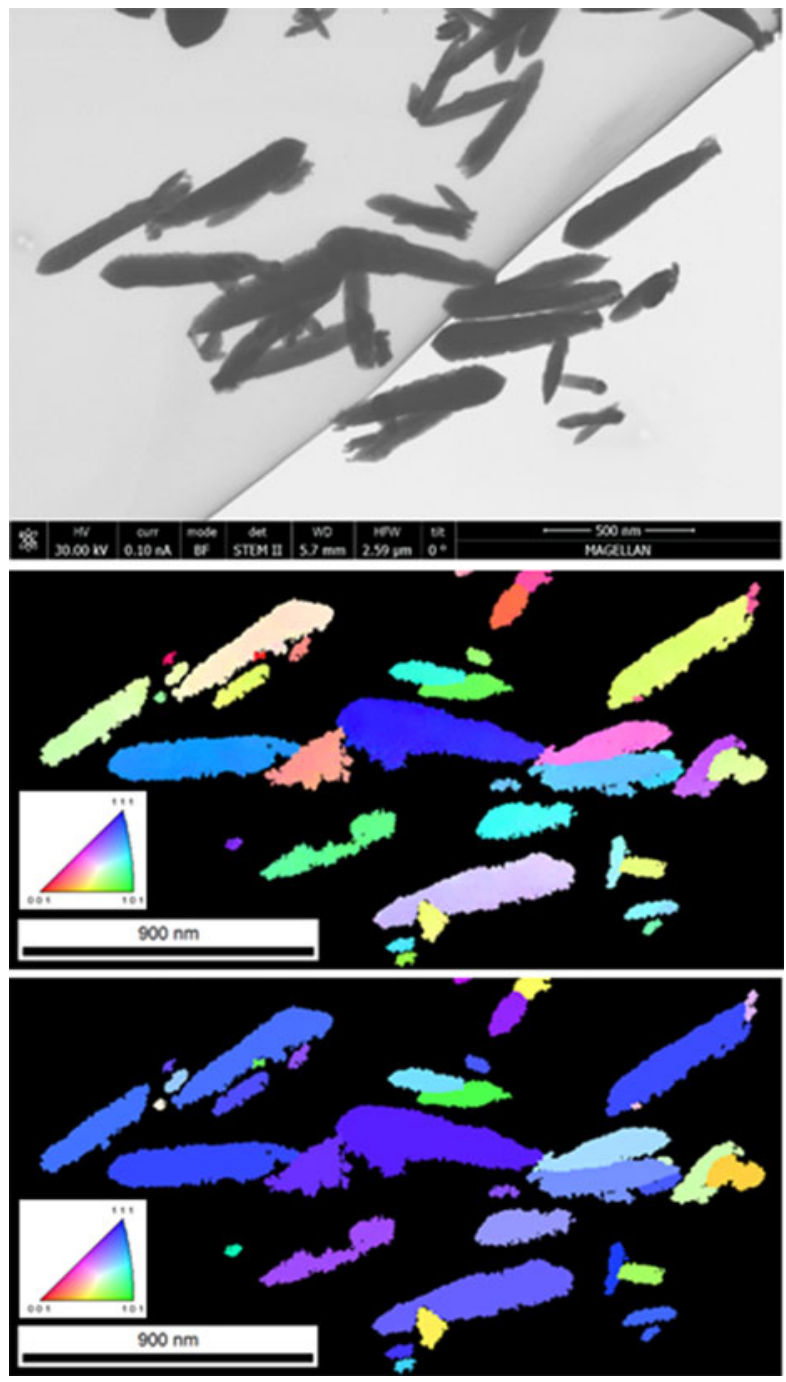

Figure 7. (Color online) $\mathrm{Fe}-\mathrm{Co}$ nano-rods dispersed on a carbon foil, imaged by STEM-BF (top) and analyzed by TKD mapping. Instead of using a conventional color code with respect to a fixed reference direction (middle), a coloring with respect to the individual grain long axis reveals their preferred $\langle 111\rangle$ orientation (bottom) (sample courtesy of Ye Tao, Zürich). with higher spatial resolution than commonly reached on bulk specimen.

\section{ACKNOWLEDGMENTS}

The access to sample material from diverse characterization projects with Urs Ramsperger, Ye Tao, Stefano Buzzi, Heinrich Siemes as well as support by Rene de Kloe (Ametek EDAX Tilburg) and Ellen Baken (FEI Eindhoven) are gratefully acknowledged.

Adams, B. L., Wright, S. I., and Kunze, K. (1993). "Orientation imaging - the emergence of a new microscopy," Metall. Trans. A - Phys. Metall. Mater. Sci. 24(4), 819-831.

Buzzi, S. (2009). Dissertation Eidgenössische Technische Hochschule ETH Zürich, http://dx.doi.org/10.3929/ethz-a-005813582.

Camus, P. P., Shapiro, J., and Prikhodko, S. V. (2011). "An EBSD study of gallium arsenide nanopillars," Mater. Sci. Forum 702-703, 916-919.

Drouin, D., Couture, A. R., Joly, D., Tastet, X., Aimez, V., and Gauvin, R. (2007). "CASINO V2.42 - a fast and easy-to-use modeling tool for scanning electron microscopy and microanalysis users," Scanning 29(3), 92-101.

Keller, R. R. and Geiss, R. H. (2012). "Transmission EBSD from $10 \mathrm{~nm}$ domains in a scanning electron microscope," J. Microsc. 245(3), 245-251.

Kunze, K., Buzzi, S., Löffler, J., and Burg, J.-P. (2008). "Benefits of Low Vacuum SEM for EBSD Applications," In EMC 2008, Aachen, Vol. 1: Instrumentation and Methods, Luysberg, M., Tillmann, K., Weirich, T., eds., pp. 575-576, DOI: 10.1007/978-3-540-85156-1_288.

Kunze, K., Sologubenko, A., Ma, H., and Spolenak, R. (2013). "Orientation contrast imaging and crystallographic orientation mapping using transmission Kikuchi diffraction in the SEM," In Proceedings Microscopy Congress 2013, Rachel, R., ed., Augsburg, pp. 744-745, urn:nbn:de: bvb:355-epub-287343.

Payton, E. J. and Nolze, G. (2013). "The backscatter electron signal as an additional tool for phase segmentation in electron backscatter diffraction," Microsc. Microanal. 19(4), 929-941.

Trimby, P. W. (2012). "Orientation mapping of nanostructured materials using transmission Kikuchi diffraction in the scanning electron microscope," Ultramicroscopy 120, 16-24.

Zaefferer, S. (2007). "On the formation mechanisms, spatial resolution and intensity of backscatter Kikuchi patterns," Ultramicroscopy 107(2-3), 254-266.
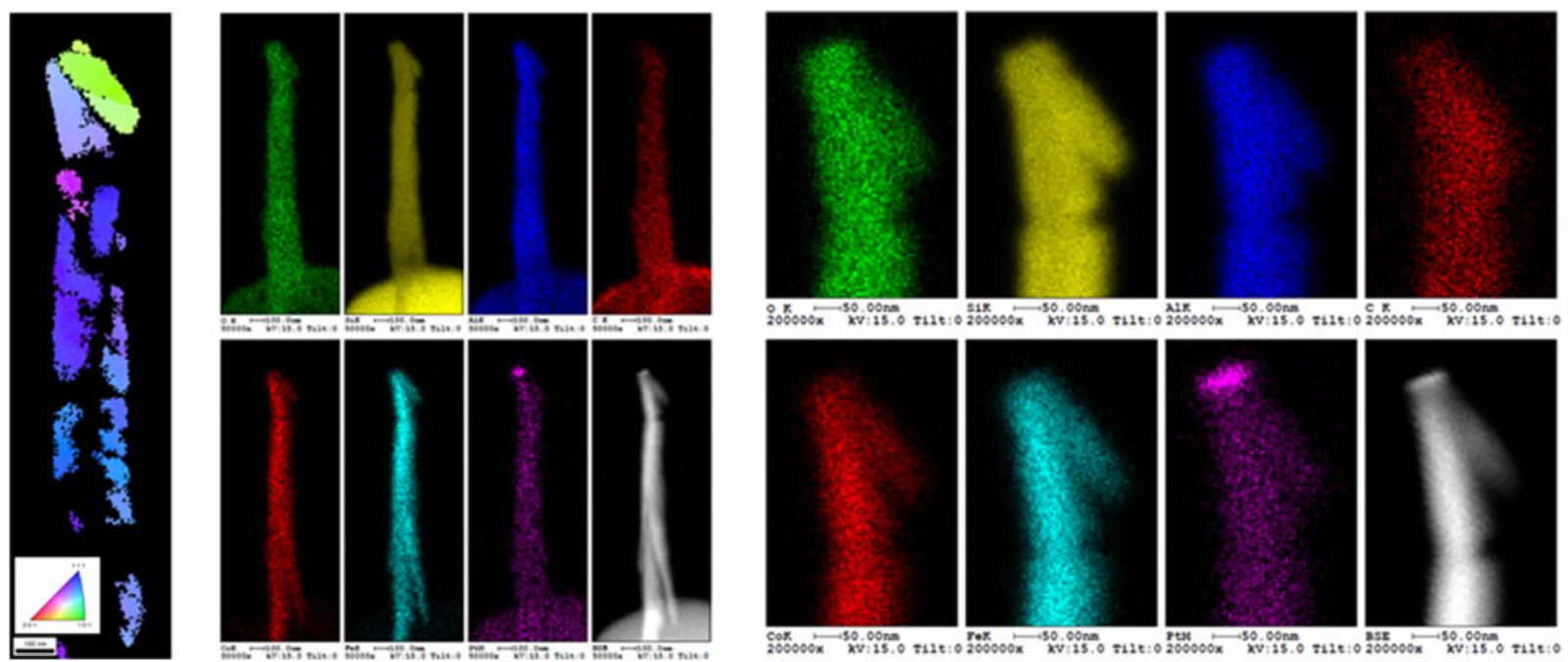

Figure 8. (Color online) Orientation map by TKD (left) and element maps by EDX (center and right) of Fe-Co nano-rods acquired in transmission geometry (FEG-SEM FEI Quanta200F at $15 \mathrm{kV}$ ). Acceptable mapping of the major elements ( $\mathrm{Fe}$ and $\mathrm{Co}$ ), but stray signals excite additional elements $(\mathrm{O}, \mathrm{Si}, \mathrm{Al}$, and C) as well. Clear detection of the seed layer $(\mathrm{Pt})$ at the top of the rod (sample courtesy of Ye Tao, Zürich). 